\title{
High-Current Neutral Electrodes
}

\author{
Definition and quality testing of high-current neutral electrodes
}

\begin{abstract}
The Standard IEC 60601-2-2:2017 refers to neutral electrodes for high current without defining them. This paper suggests a practical distinction criterion between standard and high-current neutral electrodes and a test procedure.
\end{abstract}

Keywords: Neutral electrodes, high current, long activation time, thermal features, quality test.

https://doi.org/10.1515/cdbme-2020-3159

\section{Introduction}

In electrosurgery tissue is cut and coagulated by means of high-frequency (HF) electrical current. Figure 1 shows the principle setup of a monopolar electrosurgical operation method applying HF output current to a patient via an active electrode and returning this current via a separatelyconnected neutral electrode.

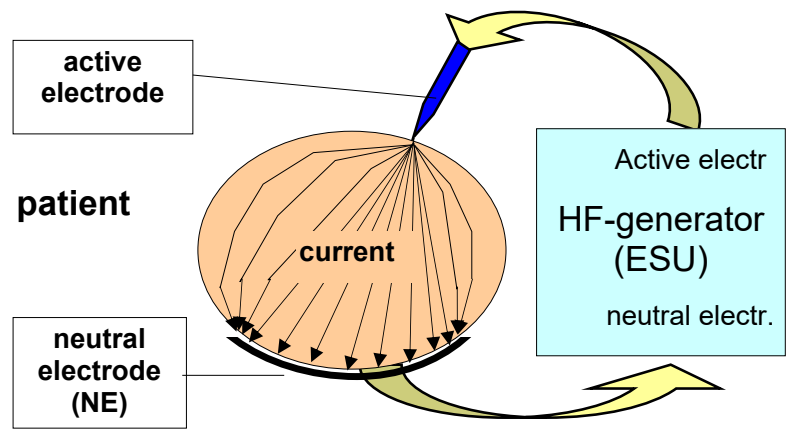

Figure 1: Principle scheme of current flow from the active electrode through the patient's body to the neutral electrode and back to the HF-generator

This operation technique is described in Standard IEC $60601-2-2,6^{\text {th }}$ Ed. 2017 [1].

\subsection{Active electrode, electrical scalpel}

The HF generator (ESU) provides the selected output power $\mathbf{P}\left[\mathbf{W}=\mathbf{V}_{\text {eff }} * \mathbf{A}_{\text {eff }}\right]$ for the surgical procedure and the surgeon calls upon this current by activating the electrical scalpel for the (short) activation time t. The energy

$$
\mathbf{E}[\mathbf{W} \mathbf{s}=\mathbf{J}]=\mathbf{P}[\mathbf{W}] * \mathbf{t}[\mathbf{s}]
$$

is transformed into heating at the electrical scalpel thus producing the desired surgical effect. The high energy density evokes local temperatures $>100^{\circ} \mathrm{C}$ so that the tissue cells burst (cut); the effect of temperatures $<100^{\circ} \mathrm{C}$ is coagulation (coag).

The procedure is visually checked by the surgeon and depends, among other things, on the preselected output $\mathbf{P}$ at the ESU and the minute duty cycle of the activation times t. According to Pearce [2] the minute duty cycle amounts to an average of $15 \%$, max. $45 \%$, which means that current only flows during, on an average, $15 \%$ of these activation times within every minute.

\subsection{Neutral electrode (NE)}

In monopolar mode the HF current is returned to the ESU via a sufficiently large neutral electrode. Whereas at the small area of the active electrode high local temperatures are necessary, the heating below the neutral electrode on the patient must not increase to a dangerous temperature. The Standard [1] limits this temperature increase to $6^{\circ} \mathrm{C}$. The energy $\mathbf{E}$ that appears at the transition resistance $\mathbf{R}$ between NE and underlying tissue, caused by the current $\mathbf{I}_{\mathbf{N E}}$ during the time $\mathbf{t}$ is calculated according to Equation (2) as follows:

$$
\mathbf{E}[\mathbf{J}]=\left(\mathbf{I}_{\mathrm{NE}}\right)^{2} * \mathbf{R} * \mathbf{t}
$$

The transition resistance $\mathbf{R}$ depends not only on size and shape of the NE, but also on the morphology and the anatomical properties of the body tissue under the NE. In [2] a heating factor $\mathbf{h f}$, independent on $\mathbf{R}$, for the energy per $\mathrm{Ohm}$ transition resistance was defined as a measure of the load on the NE as follows:

$$
\mathbf{h f}=\left(\mathbf{I}_{\mathbf{N E}}\right)^{2} * \mathbf{t}\left[\mathrm{A}^{2 * \mathrm{~s}}\right]
$$


For adult NEs, the Standard [1] defines a maximum of $30 \mathrm{~A}^{2} \mathrm{~s}$ for the energy $\mathbf{h f}$ and stipulates a temperature test on volunteers or surrogate media or with a testing device with standard load (700mA during $60 \mathrm{~s}$, corresponding to $\mathbf{3 0 A}^{\mathbf{2}} \mathbf{s}$ ) as a quality test for NEs.

\subsection{Quality tests with testing device}

The test device [3] that was developed at Innsbruck University allows to replace the elaborate tests with thermo cameras at volunteers by electronic measurements. Figure 2 shows a typical such measurement result with the test device.

Figure 3 shows a corresponding measurement made with a thermo camera on a volunteer.

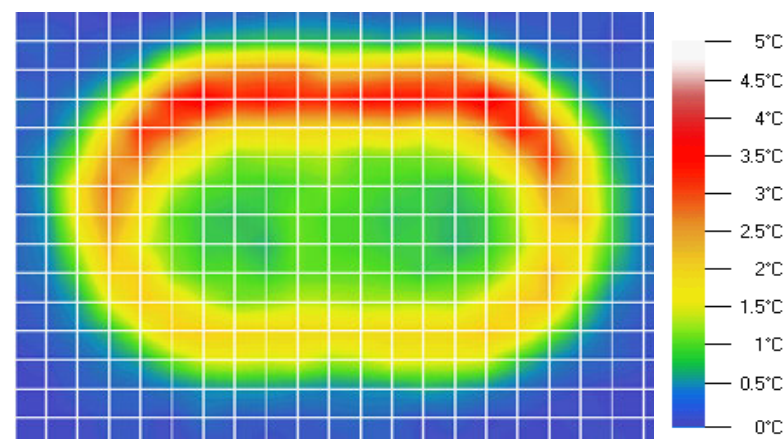

Figure. 2: Measurement under standard load with test device (electronic skin). Maximum temperature increase is $3,7^{\circ} \mathrm{C}$

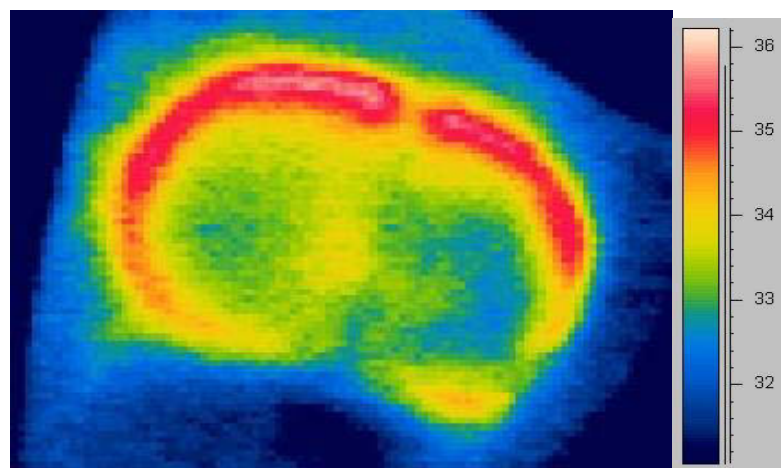

Figure. 3. Reference measurement at volunteer with thermo camera, Maximum temperature increase is $3,4^{\circ} \mathrm{C}$, $\left(32,4^{\circ} \mathrm{C}\right.$ to $\left.35,8^{\circ} \mathrm{C}\right)$

The distribution of the temperature increases an the maximum temperatures increases in Figures 2 and 3 are in good agreement and thus serve as a "calibration" of the test device [4] according to Standard [1] item 201.15.101.5.

A striking feature in both pictures is the so-called „edge effect": The highest temperature increases appear at the edges of the active area of the NE, whereas in the center area the temperature increase is lower. As is shown schematically in Fig. 1 and explained in model calculations [3], the current lines concentrate at the edge of the NE with the very edge that is closer to the site of operation showing the highest values, as was to be expected.

\section{High-current application}

The parameters $30 \mathrm{~A}^{2} \mathrm{~s}$ standard load and $700 \mathrm{~mA}$ current during 60 s are the present quality standard for NEs for socalled conventional operation techniques. Latest surgical techniques (ablation), however, apply higher currents and require longer activation times thus increasing the energy load which can no longer be safely dissipated by means of conventional NEs.

\section{1 (Hitherto) distinguishing feature}

The version of the Standard [1] presently in force defines the load (hf) for NEs in high-current mode (201.3.219) as "above" $30 \mathrm{~A}^{2} \mathrm{~s}$ in each $60 \mathrm{~s}$ interval at maximum output current and maximum duty cycle. The $6^{\circ} \mathrm{C}$ limit for the maximum temperature increase under the NE remains unchanged. This simply ascertains that conventional NEs are not suited for high-current applications, however, it does not indicate any upper limit for the energy load nor a testing procedure for high-current NEs.

Furthermore, the specification of maximum output current (201.3.226) led to misinterpretation, since a current above $700 \mathrm{~mA}$ can also occur for a short time (e.g. during "first cut") in conventional operations. As a result, even ESUs with lower output were classified as high current devices, which means that all NEs would have to belong to the high -current category of accessories.

\subsection{New approach for the distinguishing feature}

In the Technical Report [5] a number of surgical procedures are listed in Chapter 7.5.3 (Surgical procedures utilizing high currents or long duty cycles) which imply higher load for the NEs. In all cases a long activation time with a minute duty cycle of $100 \%$ is very probable. 
Instead of distinguishing by energy level (heating factor $\mathbf{h f}$ $>\mathbf{3 0 A}^{2} \mathbf{s}$ ) the new approach for the distinction between conventional und high-current surgical procedures suggests the activation time that is predetermined by the type of surgical procedure (ablation).

The different distinguishing features between conventional and high-current NEs are summarized in Table 1.

Table 1: Differences in activation times between conventional and high-current mode.

\begin{tabular}{c|c}
\hline Conventional & High current \\
\hline Heating factor $30 \mathrm{~A}^{2} \mathrm{~s}$ & Heating factor $>30 \mathrm{~A}^{2} \mathrm{~s}$ \\
\hline $\begin{array}{c}\text { Short activations within } \\
\text { every } 60 \text { s interval }\end{array}$ & $\begin{array}{c}\text { Activation time longer than } \\
60 \mathrm{~s}\end{array}$ \\
\hline Duty cycle e.g.25\% & Duty cycle $100 \%$ \\
\hline $\begin{array}{c}\text { e.g. } 15 \mathrm{~s} \text { on, } 45 \mathrm{~s} \text { off } \\
\text { or } 60 \text { s on, } 180 \mathrm{~s} \text { off }\end{array}$ & $\begin{array}{c}\text { Activation time several } \\
\text { minutes (ablation) }\end{array}$ \\
\hline
\end{tabular}

Using the activation time as a distinguishing feature would insure that already in the planning of the surgical procedure the high-current mode is recognized and the correct NEs therefore provided already at this stage.

\section{Thermal properties of high- current NEs}

Neutral electrodes for high-current mode (HC-NEs) are excluded from the tests 201.15.101.5 (NE thermal performance) and 201.15.101.9 (Monitoring NEs) described in the Standard [1]. This implies that the thermal properties of an HC-NE cannot be tested on volunteers. However, the latest version of the electronic test device [6] offers a feature to conduct thermal tests under high-current conditions.

Commercial neutral electrodes for surgery at adult patients have an active (contact) area between $90 \mathrm{~cm}^{2}$ and $160 \mathrm{~cm}^{2}$ (average value $125 \mathrm{~cm}^{2}$ ). Since neutral electrodes for high-current surgery are not as yet commercially available in the market such testing of the thermal properties at the electronic test device is conducted with an electrode of ,suitable size" of $250 \mathrm{~cm}^{2}$ (about two times the size of a conventional adult NE).

\section{Temperature increase measurements on HC-NEs}

Thermo measurements at the before described NEs with an area of $250 \mathrm{~cm}^{2}$ (HC-NE250) using currents up to 1,4A and activation times of up to $300 \mathrm{~s}(5 \mathrm{~min})$ are illustrated in Figure. 4.

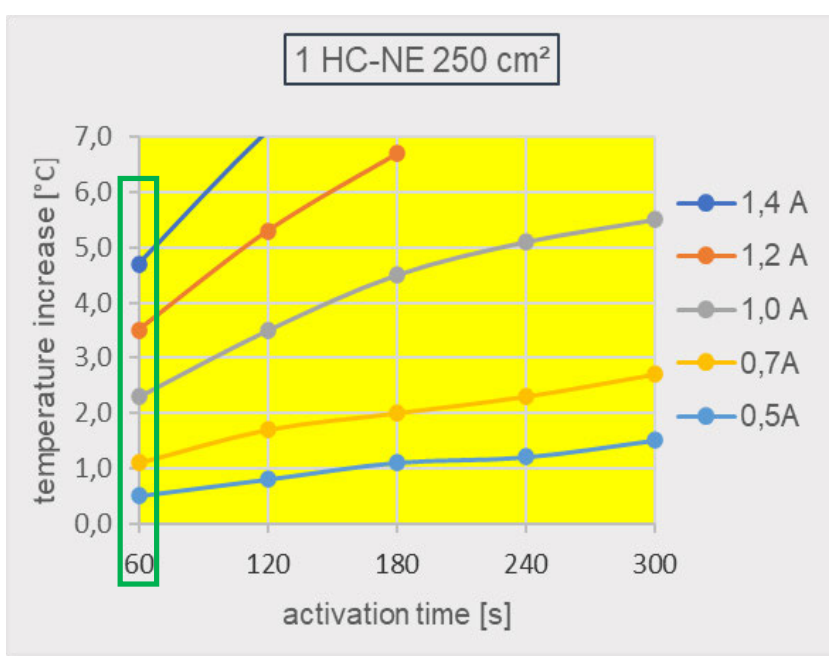

Figure. 4: Measurements of thermal features of a NE250 under high-current load with test device (electronic skin).

Green rectangle: see suggestion for a test procedure.

Table 2 gives a list of the application field (green cells) of an HC-NE for a maximum temperature increase of $6^{\circ} \mathrm{C}$ under the neutral electrode (temperatures in ${ }^{\circ} \mathrm{C}$ ).

For the currents $500 \mathrm{~mA}$ and $700 \mathrm{~mA}$ the observance of the $6^{\circ} \mathrm{C}$ limit can be estimated for activation times up to $15 \mathrm{~min}$ by linear extrapolation in the diagram Figure 4 .

Table 2: permissible range for temperature increase up to $6^{\circ} \mathrm{C}$

\begin{tabular}{|c|c|c|c|c|c|c|}
\hline $\mathrm{I}_{\mathrm{NE}} \backslash \mathrm{t}$ & $60 \mathrm{~s}$ & $120 \mathrm{~s}$ & $180 \mathrm{~s}$ & $240 \mathrm{~s}$ & $300 \mathrm{~s}$ & $\left.900 \mathrm{~s}^{*}\right)$ \\
\hline $1,4 \mathrm{~A}$ & 4,7 & 7,1 & 9,0 & & & \\
\hline $1,2 \mathrm{~A}$ & 3,5 & 5,3 & 6,7 & & & \\
\hline $1,0 \mathrm{~A}$ & 2,3 & 3,5 & 4,5 & 5,1 & 5,5 & \\
\hline $0,7 \mathrm{~A}$ & 1,1 & 1,7 & 2,0 & 2,3 & 2,7 & 5,5 \\
\hline $0,5 \mathrm{~A}$ & 0,5 & 0,8 & 1,1 & 1,2 & 1,5 & 3,2 \\
\hline
\end{tabular}

*) linear extrapolation (15 $\mathrm{min})$

It comes clear from Figure 4 that for currents between $0,5 \mathrm{~A}$ and $1,0 \mathrm{~A}$ during $300 \mathrm{~s}$ no dangerous temperature increase arises. For 1,2A and 1,4A the activations times must not exceed 150 s and 90 s, respectively and for the 
two latter energy loads 2 HC-NEs in parallel as described in Figure 5 are recommended.

Figure 5 shows the temperature increase at each of the two above mentioned HC-NEs in parallel for the indicated total current.

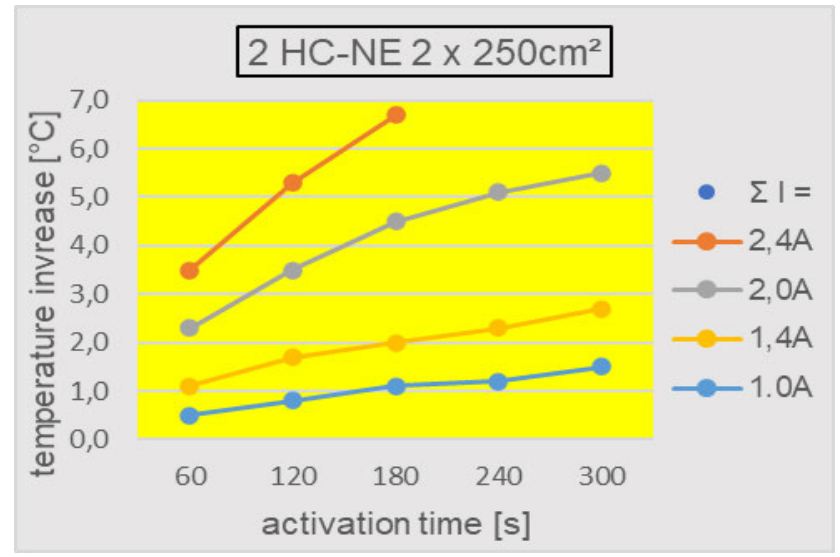

Figure. 5: Measurements of thermal features for two HC-NE250 in parallel under high current load.

Due to the new feature in the test device [6] currents up to $1,4 \mathrm{~A}$ and activations times up to $300 \mathrm{~s}$ are selectable.

For each combination of parameters as in Figures 4 and 5 the resulting "thermo pictures" are very similar to those with conventional NEs, as in Figure 2.

The measurements in Figure 4 give an indication of a test procedure that considers activation times of more than 60 seconds.

\section{Suggestion for a test procedure for HC-NEs}

There is an unpublished information by J. Eggleston [7], which forms a preliminary kind of test for HC-NEs and serves as an emergency solution until a final stipulation will be agreed: In analogy to the present quality test during 60 s with $30 \mathrm{~A}^{2} \mathrm{~s}$, as is requested by the Standard for conventional NEs, a test during $60 \mathrm{~s}$ with $120 \mathrm{~A}^{2} \mathrm{~s}$ at $6^{\circ} \mathrm{C}$ maximum admissible temperature increase is considered as the present state of the art for HC-NEs. Under this assumption the test procedure for HC-NEs is as follows:

\section{Heating factor $h f=120 A^{2} s$ Test with 1.4A during $60 \mathrm{~s}$}

Measuring a HC-NE with thermal properties other than that of the HC-NE250 at 1,4A / 60s on the test device yields a value for the maximum temperature increase $\left(\mathrm{xx}^{\circ} \mathrm{C}\right)$. If this value is entered in the diagram Figure 4 in the green rectangle (at $60 \mathrm{~s}$ ), the respective temperature curve results directly or can be interpolated.

The maximum allowable activation time can then be read from the $\mathrm{x}$-axis

If two HC-NEs are used in parallel, use figure 5 instead.

\section{References}

[1] IEC 60601-2-2, 6th Ed., 2017, Medical electrical equipment - Particular requirements for the basic safety and essential performance of high frequency surgical equipment and high frequency surgical accessories.

[2] PEARCE, JA, GEDDES, LA, VAN VLEET, JF, FOSTER, K, ALLEN, J. Skin burns from electrosurgical current. Medical Instrumentation, 1983, volume 17, number 3, pp. 225-231.

[3] Nessler N., Reischer W. Measuring device for neutral electrodes in electrosurgery, Measurement 33 (2003) pp 197-203, Elsevier Science Ltd., (www.elsevier.com).

[4] Nessler N., Reischer W., Salchner M., Calibration of the Electronic Skin, 19th International Metrology Symposium, Proceedings pp 126-128, Opatija, Croatia, 2005.

[5] IEC TR 61289 Ed2, 2019, High frequency surgical equipment and high frequency surgical accessories Operation and maintenance.

[6] Nessler N, Salchner M, Niedrist R, Verification of the thermal properties of the electronic skin, 3-Länder Tagung D-A-CH Graz, Proceedings pp 479-480, BMT 2013,

[7] unpublished information of Jeffrey L. Eggleston, heating factor for high current neutral electrodes, MT17 meeting, Edinburgh, 2014. 